\title{
Planar Angle Metrology: G-LAS, the INRIM - INFN Ring Laser Goniometer
}

\author{
Jacopo Belfi ${ }^{*}$, Nicolò Beverini ${ }^{* \dagger}$, Angela Di Virgilio ${ }^{*}$, Enrico Maccioni ${ }^{* \dagger}$, Milena Astrua ${ }^{\ddagger}$, Marco Pisani ${ }^{\ddagger}$, \\ Marco Santiano: \\ Email: \{jacopo.belfi,angela.divirgilio\}@pi.infn.it \{nicolo.beverini,enrico.maccioni\}@unipi.it \\ $\{$ m.astrua,m.pisani,m.santiano\}@inrim.it \\ *INFN, sezione di Pisa, Pisa, Italy \\ ${ }^{\dagger}$ Department of Physics, University of Pisa, Pisa, Italy \\ *Istituto Nazionale di Ricerca Metrologica, INRIM, Torino, Italy
}

\begin{abstract}
Precision mechanical industry, geodetic measurements, astronomy and experimental physics require accurate measurement of angles down to the nanoradian level. A collaboration between INRIM and INFN is developing a new transportable goniometer (G-LAS, GyroLaser Angular Standard) that makes use of a ring laser gyroscope mounted on a precision turntable with a target accuracy of $10 \mathrm{nrad}$, being the accuracy of the most precise existing angular encoders at the level of some 100 nrad. The apparatus will consist in a square optical cavity of about $0.5 \mathrm{~m}$ in side, equipped with the last generation dielectric super-mirrors that are employed in the large gyroscopes for application in seismology and geodesy.
\end{abstract}

Keywords—angular metrology; laser gyroscopes; Sagnac effect

\section{INTRODUCTION}

Ring lasers (RL) gyroscopes exploit the Sagnac effect to provide inertial measurements of rotation rate. The rotational motion of their reference frame determines a differential length of the effective optical path of the two oppositely travelling beams and by consequence a relative frequency shift (Sagnac frequency). For a ring cavity of area vector $\vec{A}$, perimeter $p$ acting at an emission wavelength $\lambda$, this frequency is

$$
f_{\text {Sagnac }}=\left|f_{C W}-f_{C C W}\right|=\frac{4 \vec{A} \cdot \vec{\Omega}}{\lambda p}=\frac{4 A}{\lambda p} \Omega \cos \theta=K \Omega
$$

being $\vec{\Omega}$ the angular speed vector of the cavity frame. This frequency difference can be efficiently observed by beating on a photodetector the two beams emitted from the laser cavity [1]. The factor $K$ (i.e. the ratio between the angular speed and the Sagnac frequency) is defined as the RL gyroscope sensitivity factor. Laser gyroscopes with perimeter-length of tens of centimetres are commonly used for inertial navigation applications while larger systems (side-length larger than one meter), rigidly fixed to the ground, provide precise measurements of the Earth rotation rate and of the small superimposed local rotations coming from geophysical and geodetic phenomena.

Applications of RL's in Angular Metrology have been foreseen since the end ' $60 \mathrm{~s}$. The basic idea is to use the interference fringes by the two counter-propagating modes as an ultra-fine angular scale dividing the full angle into a number of $N=P / \lambda$ intervals. The most effective realization of such kind of goniometer is the apparatus developed by $\mathrm{Yu}$. V. Filatov and collaborators since the end of '70s. It consists in a monolithic $\mathrm{RL}, 11 \mathrm{~cm}$ in side-length, mounted on a turntable. The RL is equipped with total reflection prisms in optical contact with a Zerodur cavity frame [2]. The typical resolution of this instrument is at the level of $100 \mathrm{nrad}$, limited by the errors due to the influence of environmental parameters on the ring laser dynamics.

In this paper we will describe the state-of-art of G-LAS, the prototype of a transportable goniometer based on a RL that is under construction at the Italian National Institute for Metrologic Research (Istituto Nazionale di Ricerca Metrologica - INRIM) with the collaboration of the Italian Institute for Nuclear Physics (Istituto Nazionale di Fisica Nucleare - INFN).

This project exploits the large expertise on RL gyroscope acquired in the recent years by the group operating at the section of Pisa of INFN and at the Pisa University Department of Physics [3,4] with the purpose of building a gyroscope having the very high sensitivity that needs for detecting in a terrestrial laboratory the thin effect foreseen by the general relativity equations, due to the distortion of the space-time metrics induced by the rotating Earth mass (the so called Lense-Thirring effect), which is expected to give a correction of the order of $1 \mathrm{ppb}$ over the Earth rotation speed [5,6].

Precision mechanical industry, geodetic measurements, astronomy and experimental physics require accurate measurement of angles down to the nanoradian level. The target of the present prototype is to build a goniometer with an angular accuracy of the order of 0.002 arcsec (equivalent to 10 nrad), being the accuracy of the most precise angular encoders working in the world at the level of some $100 \mathrm{nrad}$ in spite of a resolution more than one order of magnitude better.

\section{THE RL GONIOMETERS}

The realization of the angular scale is made by subdividing the circle into a number of equal intervals in fact implemented with the circular grating of angle encoders. The resolution in the encoder performance in the present state-of-art measurement apparatus has achieved nanoradian level, but the accuracy is limited by technological factors to the microradian 
level. Absolute calibration of such instruments is today an open issue of angle metrology.

A RL mounted on a continuously rotating table can act as an ideal angular encoder. The measure of a rotation angle $\phi$ can be evaluated as $\phi=2 \pi N_{\phi} / N_{2 \pi}$, being $N_{\phi}$ and $N_{2 \pi}$ the number of fringes counted by beating the two counter-rotating laser beams, respectively, when the table rotates by $\phi$ and the full $2 \pi$ angle.

As a fact, the number of fringes observed in the time $t_{1}-t_{0}$ when the table rotates by an angle $\phi$ is:

$$
N_{\phi}=\frac{1}{2 \pi} \int_{t_{0}}^{t_{1}} f(t) d t=\frac{K}{2 \pi} \int_{t_{0}}^{t_{1}} \Omega(t) d t=\frac{K}{2 \pi} \phi
$$

while the number observed in the period $T$, correspondent to a full a $2 \pi$ rotation is:

$$
N_{2 \pi}=\frac{1}{2 \pi} \int_{t_{0}}^{t_{0}+T} f(t) d t=\frac{K}{2 \pi} \int_{t_{0}}^{t_{0}+T} \Omega(t) d t=K
$$

By (3) it appears that RL sensitivity factor $K$ can be accurately determined each time a measure is performed, providing an accurate self calibration of the apparatus without any a priori knowledge of the exact geometry of the instrument.

While angle encoders have very high sensitivity, RL has high intrinsic accuracy. A transportable RLG goniometer can consequently provides an ideal apparatus to make the intercalibration of encoders in use in the different metrological Institutes.

\section{ACCURACY OF RL GONIOMETER}

In a previous paper [7] we presented a simplified analysis of the different sources of inaccuracy that can affect a RL goniometer. A more complete analysis can be found in [8].

Up today, the only existing goniometer based on RL gyroscope is the apparatus EUP-1L, developed at the St. Petersburg Electrotechnical University [2]. The ring laser consists in a Zerodur monolithic cavity $44 \mathrm{~cm}$ in perimeterlength, equipped with total reflection prisms in optical contact with the cavity frame [9]. The cavity is filled by a He-Ne mixture and is lasing on the classical red line at $632.8 \mathrm{~nm}$. The laser is fixed on a turntable, and a counter measures the number of fringes corresponding to the rotation angles. Specimens of EUP-1L are in use in some metrological institutions in the world. Its typical resolution is limited to 0.1 " $(0.5 \mu \mathrm{rad})$ by the influence of the environmental parameters on the ring laser dynamics (birifrangency induced by spurious magnetic fields on the corner prisms, mechanical instabilities of the rotator, non-optimization of the geometrical parameters of the laser cavity). The resolution of the prototype active in St. Petersburg has been pushed down to 100 nrad through a careful (and quite cumbersome) analysis of all these systematic errors [10].

Finally, the performances are limited by the RL dimension. Statistical noise on the angle definition is dominated by shot noise. In a RL with the optical cavity quality factor $Q$ for a single measurement it is given by:

$$
\delta \Omega_{\text {shot noise }}=\frac{c}{L Q} \sqrt{\frac{h v \tau}{P_{\text {out }}}}
$$

where $P_{\text {out }}$ is the detected radiation power, $c$ is the light speed, and $\tau$ is the measuring time, which corresponds to the period of the turntable. Thanks to the fact that the scale constant $K$ is self-calibrated every complete turn of the table, quantum noise can be further reduced averaging over many measurements.

Both the ratio $A / p$ and $Q$ increase linearly with the side dimension. Thus, increasing the RLG dimension can be very effective in reducing shot noise. Considering a mirror reflectivity of the order of $99,999 \%, \tau \approx 5 \mathrm{~s}$, and typical value of laser operation, the shot noise in a single measurement amounts to $330 \mathrm{nrad} / \mathrm{s}$ for a square ring cavity with side $L=11 \mathrm{~cm}$ (as in Filatov's instruments), while is $15 \mathrm{nrad} / \mathrm{s}$ for $L=50 \mathrm{~cm}$ (as in present prototype).

Other possible sources of errors are related to change in the scale factor $K$ during the measuring time $\tau$ (around $10 \mathrm{~s}$ ). An accuracy target of 0.002 arcsec (equivalent to $10 \mathrm{nrad}$ ) requires a temperature stability of the structure of a few $\mathrm{mK}$. Also the wobble of the rotating table and the rotational speed stability must be controlled at $10^{-4}$ level. Moreover, (1) is only a first order approximation of the actual relation between the observed Sagnac frequency and the rotational speed. In a more complete analysis, it is necessary to take into account the coupling between the two counter-propagating laser beams due to the back-scattering on the mirrors and the possible effect of non-reciprocity in the two optical paths. Also these effects are strongly reduced by enlarging the RL dimensions.

From all these considerations it is evident that larger ring dimensions will provide better performances. But this has a price: longer optical path means smaller free spectral range of the optical cavity that eventually become smaller then the laser homogeneous linewidth, making more and more difficult to achieved stable single longitudinal mode laser operation. The experience with large frame RL demonstrates that this problem can be partially bypassed by increasing the pressure of the gas in the active medium (then, enlarging the homogeneous linewidth by pressure broadening) and by operating the laser very close to its threshold. Moreover, when the target is to build a transportable instrument, weight and dimensions

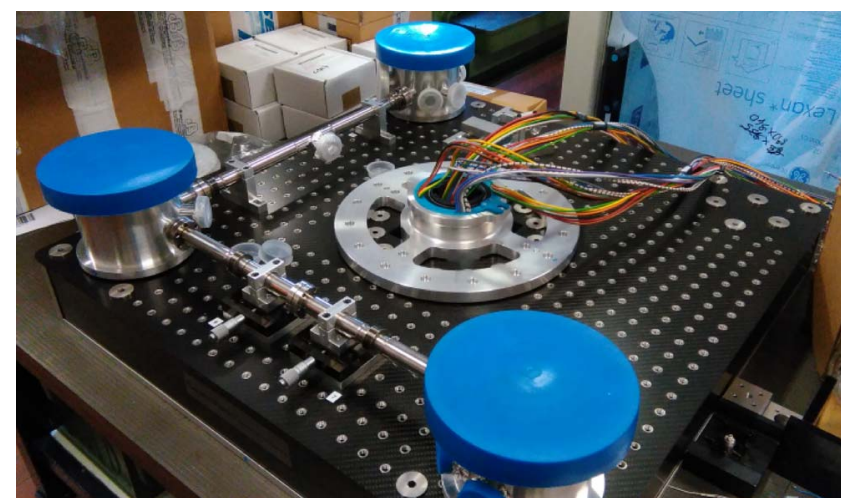

Fig. 1. Picture of the carbon fibre board mounted on the turntable. Three corner tower and two sides of the ring vacuum chamber has been already fixed on the board. 


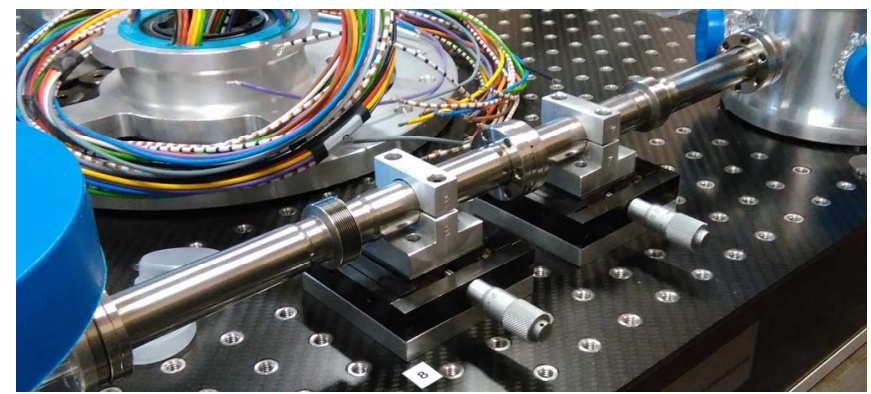

Fig. 2. The sliding stages to position the diaphragm. The diaphram is inserted in the gasket connecting the two ConFlat flanges between the two stages.

become obviously critical parameters.

\section{THE INRIM-INFN GONIOMETER}

The design of our RL goniometer is based on a square RL mounted on a precision turntable. The optical cavity is delimited by the four mirrors, without any physical confinement of the active medium on the optical path, so that the intra-cavity losses are limited only by mirrors reflectivity. Then, all the cavity is filled with the He-Ne mixture.

The ring optical cavity, with the side of $50 \mathrm{~cm}$, is mounted on a $90 \times 90 \mathrm{~cm}^{2}$ board realized by a carbon fibre honeycomb, closed between two layers of carbon fibre panels. Board material had chosen for its properties of stiffness and lightness and for its moderate thermal expansion coefficient $\left(2.5 \cdot 10^{-6}\right.$ $\left.\mathrm{K}^{-1}\right)$. The board is fixed on one side to the rotating table and on the other side to the ring laser vacuum chamber through stainless steel inserts. (Fig. 1). The vacuum chamber consists in four corner towers, connected together by pipes enclosing the optical path. Thermal stability of the cavity must be provided by the carbon fibre board. Bellows provides the needed elasticity to the connections of the pipes to the towers. In the middle of one of the pipes is joint a pyrex capillary, where external electrodes can excite by radiofrequency the laser discharge. The four angular towers host the mirrors with their mechanical mounting and present openings for electrical feedthroughs, windows for the output of the single beam and interference signal and for visual inspection. To reduce the weight of the structure, they are worked on Aluminium that is much lighter than the usual stainless steel. The connections between the vacuum chamber components are all realized with UHV ConFlat standard. A valve allows the connection to the vacuum pumps and to the gas reservoir for initial operation of filling the He-Ne mixture. A getter pump keeps the purity of the gas in the time.

The turntable, produced by Aerotech (model ABRS$300 \mathrm{MP}$ ), is an air bearing, with integrated direct driving motor and high accuracy optical encoder. By the high accuracy manufacturing it achieves sub-microradian rotation errors and a resolution in angle position better than $0.2 \mu \mathrm{rad}$. The most important feature for our application is that the table is capable of rotating at a constant speed $\Omega$ with a relative error $\Delta \Omega / \Omega$ of the order of $10^{-5}$. A hole placed in the centre of the table allows inserting a sliding contact assembly used to connect the RL signals, controls and power supplies to the laboratory.

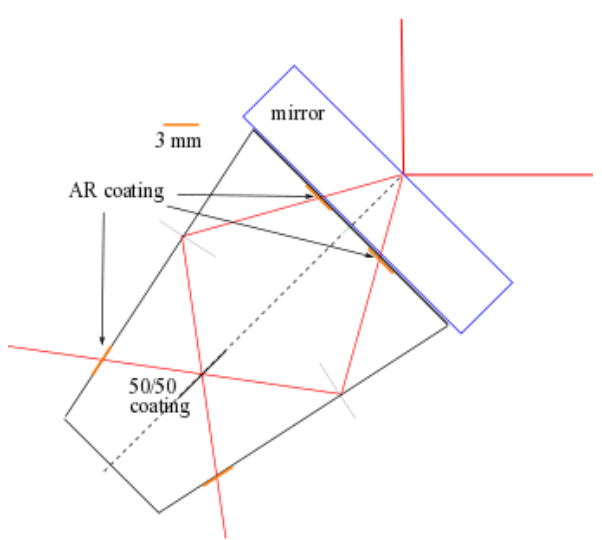

Fig. 3. The combining prism (Koester prism). It consists in two symmetric quartz prism glued together. On the contact region of the two prisms a semitransparent 50/50 coating allows beams combining

The optical cavity is closed by two flat and two concave mirrors. The radius of the concave mirrors is $4 \mathrm{~m}$. The pipe with the discharge is placed between the two concave mirrors. With this geometry, the optical cavity is stable, with a waist in the discharge region and one in the middle of the opposite side with the dimensions ( 4 times the $1 / \mathrm{e}^{2}$ beam intensity radius) of $2.17 \times 2.41 \mathrm{~mm}^{2}$ and $1.64 \times 2.17 \mathrm{~mm}^{2}$, respectively. In order to favour laser operation on TEM00 mode, a diaphragm will be inserted in the pipe at the second waist (Fig. 2). The exact

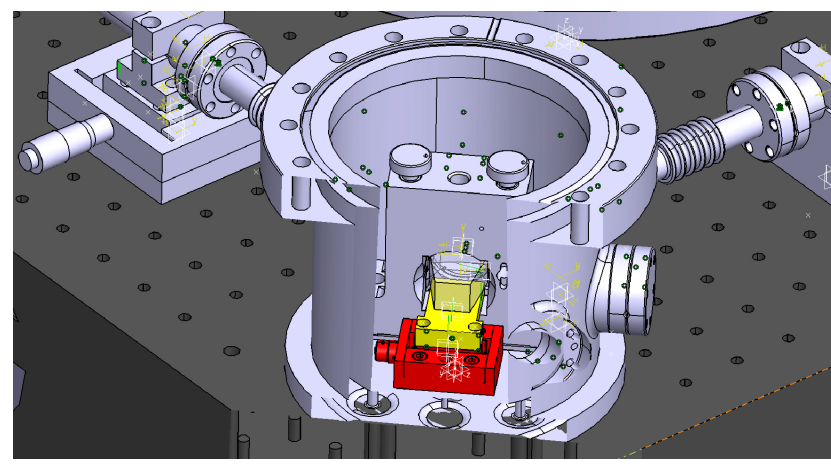

Fig. 4. Sketch of the corner tower accommodating a curved mirrors and the combiner prism. The mechanical and the sliding share stage are highlighted in yellow and in red, respectively,

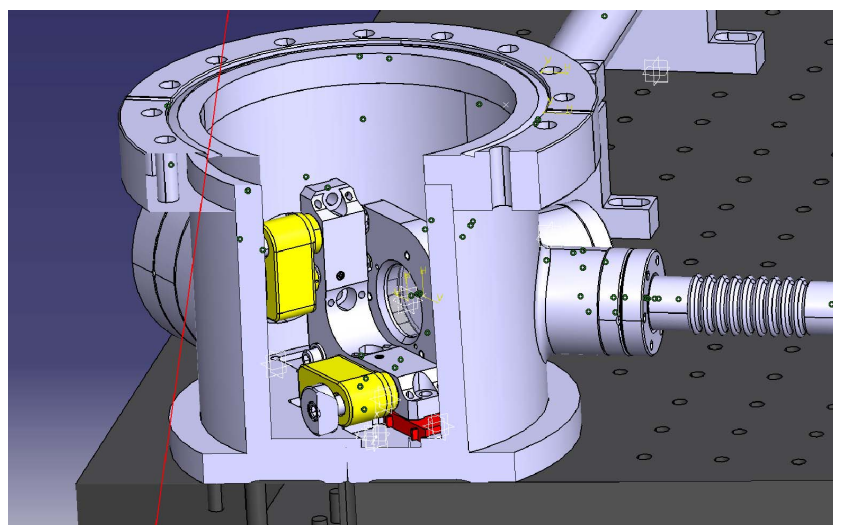

Fig. 5. Sketch of one corner tower that accommodate the flat mirrors. The motorized tilt controls and the sliding share stage are highlighted in yellow and in red, respectively, 
position of the diaphragm will be adjusted by moving the pipe through linear travel stages. The mirrors are top-quality mirrors, with the best available surface roughness $(<1 \AA)$ and a reflectivity R near to $99.999 \%$, in order to increase the cavity quality factor and to minimize the coupling between the counter-rotating traveling beams due to backscattering. Beside one of the two concave mirrors, a specially designed prism (Koester prism) combines the two counter-rotating laser beams (Fig. 3 and 4). The interference signal exits the vacuum chamber through a window and is detected by a photodiode. The laser beams transmitted by the second concave mirror are detected by other photodiodes for the diagnostics of the laser operation and to provide the stabilization of the laser wavelength. The two flat mirrors orientation can be fine adjusted under vacuum by motorized tilt regulations, while PZT driven linear share stages can shift the mirrors in the direction orthogonal to its surface (Fig. 5).

The mirror's coating is extremely delicate and requires the respect of a high cleanness level. Then the mirrors will be mounted inside the structure, operating in clean room. There, we will perform also the rough alignment of the optical cavity, adjusting the mirror mechanical controls and the combiner prism with the help of an auxiliary external green laser beam injected through a mirror. The laser chamber is then filled with the He-Ne gas mixture and is sealed off, closing the valve of connection with the vacuum system. A getter pump, which is active for molecular gases and not to noble gases, keeps the purity of the mixture in the time. Further fine alignment will be performed through the motorised controls of the flat mirrors. For a reasonable range of perturbation, this set of control is able to fully control the optical cavity. In particular the system is robust against thermal dilatations that will not affect the alignment of the two curved mirrors, the discharge tube and the combiner prism.

The active medium is excited by a radiofrequency discharge through external electrodes. Radiofrequency excitation is chosen to avoid plasma cataphoresis that would perturb the Sagnac signal. As stated before, the RL will operates near to threshold, in order to have stable operation condition without mode jumping. For this purpose, it is important to stabilise the optical cavity length, keeping the laser emission wavelength on the maximum of the gain profile. Thermal optical cavity shift can be corrected acting on the PZT stages translating the flat mirrors. Moving the two mirrors together, the length of the two sides of the ring that connect the concave to the flat mirrors will change of the same amount.
This operation does not affect neither the position of the beam spot on the two curved nor the alignment of the discharge capillary and of the combiner prism. The stabilisation of the optical length to the maximum laser output power will be implemented by a servo lock, superimposing a small sinusoidal dithering on the PZT drivers voltage and detecting in-phase on a photodiode the single beam output.

\section{ACKNOWLEDGMENTS}

We have to thank Filippo Bosi for its support in the use of the clean room facility. We thanks also dr. Giorgio Carelli and dr. Andreino Simonelli for the useful discussions.

Present project is funded by the Italian Ministero dell'Istruzione, dell'Università e della Ricerca in the frame of "Progetti premiali".

\section{REFERENCES}

[1] K.U. Schreiber, and J-P. R. Wells, "Large ring lasers for rotation sensing”, Rev Sci Instrum vol. 84 041101, 2013

[2] Yu. V. Filatov, D. P. Loukianov and R. Probst, "Dynamic angle measurement by means of a ring laser ", Metrologia vol. 34 pp. 343-351, 1997.

[3] J. Belfi, N. Beverini, F. Bosi, G. Carelli, A. Di Virgilio, E. Maccioni, A. Ortolan, and F. Stefani: "A $1.82 \mathrm{~m}^{2}$ ring laser gyroscope for nanorotational motion sensing”, Appl Phys B vol. 106, pp.271-281, 2012

[4] J.Belfi, et al.: "Performance of G-Pisa ring laser gyro at the Virgo site", J. Seism. vol. 16, pp. 757-766. 2012

[5] F. Bosi, et al., "Measuring Gravito-magnetic Effects by Multi RingLaser Gyroscope", Phys Rev D vol. 84 122002, 2011

[6] A. Di Virgilio, et al.: "A ring lasers array for fundamental physics", C. R. Physique vol. 15, pp. 866-874, 2014

[7] M. Astrua, J. Belfi, N. Beverini, A. Di Virgilio, G. Carelli, E. Maccioni, A. Ortolan, M. Pisani, and M. Santiano The INRIM - INFN ring laser gyroscope for planar angle metrology application, Fotonica 2015 Proceedings, AEIT Italian Conference on Photonics Technologies, Torino, 6-8 May 2015

[8] V.E. Primalov and Yu. V. Filatov, "Investigation of the output characteristic of a rotating ring gas laser", Sov. J. QE vol. 7 pp. 802-806, 1977.

[9] E. N. Zhuravleva, V. N. Kuryatov, and B. N. Semenov, 2nd Saint Petersburg Int. Conf. Gyroscopic Technology and Navigation, Part 2, St Petersburg, SCRI 'Electropribor', pp. 57-59, 1995.

[10] M. N. Burnashev, P. A. Pavlov, and Yu. V. Filatov, "Development of precision laser goniometer systems", Quantum Electronics vol. 43 pp. $130-138,2013$. 